\title{
THE HETEROGENEOUS STRING: COUPLED HELICES IN HILBERT SPACE*
}

BY

J. T. LEWIS

Dublin Institute for Advanced Studies

1. We solve the initial-value problem for the partial-differential equation

$$
u_{t t}-s(x)^{2} u_{x x}=0 \text {, }
$$

given

$$
u(x, 0)=f(x), \quad u_{t}(x, 0)=g(x)
$$

in matters of notation, we follow [1]. We take as the phase-space for the problem the space of initial data having finite energy, with inner product derived from the energy quadratic form. As in [1], we first straighten the characteristics of (1.1) with the transformation

$$
x \mapsto y(x)=\int_{0}^{x} d z / s(z) ;
$$

we have, from (1.1), the equation

$$
u_{t t}-u_{y y}+\left(c_{y} / c\right) u_{y}=0, \quad c(y(x))=s(x) .
$$

The energy $E$ is given by

$$
E=\frac{1}{2} \int_{-\infty}^{\infty}\left(u_{y}^{2}+u_{t}^{2}\right) d y / c(y) .
$$

Taking a point $v$ in phase-space to be a pair

$$
v=\left|\begin{array}{l}
q \mid \\
|p|
\end{array}\right|, \quad q(y)=u_{y}(y, 0), \quad p(y)=u_{t}(y, 0),
$$

the inner product is given by

$$
\left\langle v_{1}, v_{2}\right\rangle=\left(C^{-1} v_{1}, C^{-1} v_{2}\right), \quad C=\left(\begin{array}{cc}
c(x)^{1 / 2} & 0 \\
0 & c(x)^{1 / 2}
\end{array}\right)
$$

and

$$
\left(v_{1}, v_{2}\right)=\frac{1}{2} \int_{-\infty}^{\infty}\left(q_{1}(y) q_{2}(y)+p_{1}(y) p_{2}(y)\right) d y .
$$


The propagation of a disturbance according to Eq. (1.4) induces a flow on phasespace; since energy is conserved, the flow preserves the inner product $\langle\cdot, \cdot\rangle$ and there is at most one trajectory through each point of phase-space. From (1.4), the equation governing the flow is

$$
\frac{d}{d t} v(t)=L v(t)
$$

where

$$
L=\left(\begin{array}{cc}
0 & \partial_{y} \\
\partial_{y}-2 \gamma & 0
\end{array}\right), \quad 2 \gamma(y)=c^{\prime}(y) / c(y) .
$$

The generator $L$ is skew-symmetric with respect to the energy inner product (1.7) and so there exists a one-parameter group $V(t)$ of orthogonal transformations determined by

$$
\frac{d}{d t} V(t)=L V(t), \quad V(0)=1,
$$

so that $v(t)=V(t) v$ solves (1.9). The problem is to find a useful expression for $V(t)$.

2. By a sequence of linear transformations we reduce $V(t)$ to a perturbation of a flow which is easy to compute, so that we get a perturbation series for $V(t)$. The method has the advantage that the terms in the perturbation series have an intuitive interpretation: this makes it easy to write them down from memory. Put

$$
L=R C^{-1} L C R^{-1}, \quad \tilde{V}(t)=e^{t L}=R C^{-1} V(t) C R^{-1},
$$

where $C$ is given by (1.7), and

$$
R=\left(\begin{array}{rr}
2^{-1 / 2} & -2^{1 / 2} \\
2^{-1 / 2} & 2^{1 / 2}
\end{array}\right)
$$

Then we have

$$
\tilde{L}=\tilde{L}_{0}+\Gamma, \quad \tilde{L}_{0}=\left(\begin{array}{rc}
-\partial y & 0 \\
0 & \partial y
\end{array}\right), \quad \Gamma=\left(\begin{array}{cr}
0 & -\gamma \\
\gamma & 0
\end{array}\right) .
$$

Putting

$$
\tilde{U}(t)=e^{t \tilde{L}_{0}}
$$

we see easily that

$$
\tilde{U}(t)=\left(\begin{array}{cc}
T(t) & 0 \\
0 & T^{\dagger}(t)
\end{array}\right),
$$

where $T(t)$ is right-translation by $t$,

$$
(T(t) f)(s)=f(s-t)
$$

and $T^{\dagger}(t)$ is left-translation by $t$,

$$
T^{\dagger}(t)=T(-t)
$$


The well-known perturbation formula

$$
\tilde{V}(t)=\tilde{U}(t)+\int_{0}^{t} \tilde{U}(t-s) \Gamma \tilde{V}(s) d s
$$

(see [4], p. 497) then yields an infinite series for $\tilde{V}(t)$ through the iteration scheme

$$
\tilde{V}^{(n+1)}(t)=\tilde{U}(t)+\int_{0}^{t} \tilde{U}(t-s) \Gamma \tilde{V}^{(n)}(s) d s, \quad \tilde{V}^{(0)}(t)=\tilde{U}(t) .
$$

We can interpret all this as follows: phase-space decomposes into a pair of complementary subspaces; this induces a decomposition of each initial datum into a forwardpropagating part and a backward-propagating part. Indeed, in the homogeneous case $(\Gamma=0)$ they propagate independently, since then $\tilde{V}(t)=\tilde{U}(t)$. In the heterogeneous case they are coupled; we choose to regard the coupling $\Gamma$ as a perturbation. We gain further understanding by writing $\tilde{V}(t)$ in block form:

$$
\tilde{V}(t)=\left(\begin{array}{ll}
\tilde{V}_{\mathrm{FF}}(t) & \tilde{V}_{\mathrm{FB}}(t) \\
\tilde{V}_{\mathrm{BF}}(t) & \tilde{V}_{\mathrm{BB}}(t)
\end{array}\right)
$$

Here the term $\tilde{V}_{\mathrm{FF}}(t)$ maps forward-going data into forward-going data, $\tilde{V}_{\mathrm{FB}}(t)$ maps backward-going data into forward-going data, and so on. From (2.5) and (2.9) we have

$$
\begin{gathered}
\tilde{V}_{\mathrm{FF}}(t)=T(t)-\int_{0}^{t} \int_{0}^{t_{1}} T\left(t-t_{1}\right) \gamma T^{\dagger}\left(t_{1}-t_{2}\right) \gamma T\left(t_{2}\right) d t_{1} d t_{2}+\cdots, \\
\tilde{V}_{\mathrm{FB}}(t)=\int_{0}^{t} T^{\dagger}\left(t-t_{1}\right) \gamma T\left(t_{1}\right) d t_{1}-\int_{0}^{t} \int_{0}^{t_{1}} \int_{0}^{t_{2}} \\
\times T^{\dagger}\left(t-t_{1}\right) \gamma T\left(t_{1}-t_{2}\right) \gamma T^{\dagger}\left(t_{2}-t_{3}\right) \gamma T\left(t_{3}\right) d t_{1} d t_{2} d t_{3}+\cdots, \\
\tilde{V}_{\mathrm{BF}}(t)=-\int_{0}^{t} T\left(t-t_{1}\right) \gamma T^{\dagger}\left(t_{1}\right) d t_{1}-\int_{0}^{t} \int_{0}^{t_{1}} \int_{0}^{t_{2}} \\
\times T\left(t-t_{1}\right) \gamma T^{\dagger}\left(t_{1}-t_{2}\right) \gamma T\left(t_{2}-t_{3}\right) \gamma T^{\dagger}\left(t_{3}\right) d t_{1} d t_{2} d t_{3}+\cdots, \\
\tilde{V}_{\mathrm{BB}}(t)=T^{\dagger}\left(t_{1}\right)-\int_{0}^{t} \int_{0}^{t_{1}} T^{\dagger}\left(t-t_{1}\right) \gamma T\left(t_{1}-t_{2}\right) \gamma T^{\dagger}\left(t_{2}\right) d t_{1} d t_{2}+\cdots
\end{gathered}
$$

Consider the terms in the series (2.11a) for $\tilde{V}_{\mathrm{FF}}(t)$ : the first term simply translates a forward-going datum in the forward direction. The second term (2.11a) is a correction of order $\gamma^{2}$; the integrand

$$
T\left(t-t_{1}\right) \gamma T^{\dagger}\left(t_{1}-t_{2}\right) \gamma T\left(t_{2}\right)
$$

translates a forward-going datum in the forward direction from time zero to time $t_{2}$ when it is reflected. On reflection it is multiplied by the local reflection coefficient $\gamma$, then translated backwards from time $t_{2}$ to time $t_{1}$, when it is reflected again, multiplied by $\gamma$ and translated forwards from time $t_{1}$ to time $t$. The integrated term represents the contribution to the forward-going disturbance from all possible double reflections. There is a similar interpretation of all the terms in the series $(2.11 \mathrm{a}-\mathrm{d})$ which makes them easy to write down from memory. 
3. It remains to put the pieces together again; from (2.1) we have

$$
V(t)=C R^{-1} \tilde{V}(t) R C^{-1} .
$$

Using (2.10) and (2.11), we have

$$
\begin{aligned}
& V(t)= \\
& \left(\begin{array}{ll}
\frac{1}{2} c^{1 / 2}\left(V_{\mathrm{FF}}(t)+V_{\mathrm{BB}}(t)+V_{\mathrm{FB}}(t)+V_{\mathrm{BF}}(t)\right) c^{-1 / 2} & \frac{1}{2} c^{1 / 2}\left(V_{\mathrm{BB}}(t)-V_{\mathrm{FF}}(t)+V_{\mathrm{FB}}(t)-V_{\mathrm{BF}}(t)\right) c^{-1 / 2} \\
\frac{1}{2} c^{1 / 2}\left(V_{\mathrm{BB}}(t)-V_{\mathrm{FF}}(t)+V_{\mathrm{BF}}(t)-V_{\mathrm{FB}}(t)\right) c^{-1 / 2} & \frac{1}{2} c^{1 / 2}\left(V_{\mathrm{FF}}(t)+V_{\mathrm{BB}}(t)-V_{\mathrm{FB}}(t)-V_{\mathrm{BF}}(t)\right) c^{-1 / 2}
\end{array}\right) .
\end{aligned}
$$

Thus $u_{t}(y, t)$ is given in terms of the initial data

$$
u(y, 0)=\varphi(y), \quad u_{t}(y, 0)=\psi(y),
$$

by

$$
\begin{aligned}
u_{t}(y, t)= & \frac{1}{2} c(y)^{1 / 2}\left(V_{\mathrm{BB}}(t)-V_{\mathrm{FF}}(t)+V_{\mathrm{BF}}(t)-V_{\mathrm{FB}}(t)\right)\left(c^{-1 / 2} \varphi^{\prime}\right)(y) \\
& +\frac{1}{2} c(y)^{1 / 2}\left(V_{\mathrm{BB}}(t)+V_{\mathrm{FF}}(t)-V_{\mathrm{BF}}(t)-V_{\mathrm{FB}}(t)\right)\left(c^{-1 / 2} \psi\right)(y),
\end{aligned}
$$

so that

$$
\begin{aligned}
u(y, t)=\varphi(y) & +\frac{1}{2} c(y)^{1 / 2} \int_{0}^{t}\left[V_{\mathrm{BB}}\left(t_{1}\right)-V_{\mathrm{FF}}\left(t_{1}\right)+V_{\mathrm{BF}}\left(t_{1}\right)-V_{\mathrm{FB}}\left(t_{1}\right)\right]\left(c^{-1 / 2} \varphi^{\prime}\right)(y) d t_{1} \\
& +\frac{1}{2} c(y)^{1 / 2} \int_{0}^{t}\left[V_{\mathrm{FF}}\left(t_{1}\right)+V_{\mathrm{BB}}\left(t_{1}\right)-V_{\mathrm{BF}}\left(t_{1}\right)-V_{\mathrm{FB}}\left(t_{1}\right)\right]\left(c^{-1 / 2} \psi\right)(y) d t_{1} .
\end{aligned}
$$

When $c^{\prime}(y)$ is identically zero this reduces to

$$
u\left(y_{1} t\right)=\varphi(y)+\frac{1}{2} \int_{0}^{t}\left[T^{\dagger}\left(t_{1}\right)-T\left(t_{1}\right)\right] \varphi^{\prime}(y) d t_{1}+\frac{1}{2} \int_{0}^{t}\left[T^{\dagger}\left(t_{1}\right)+T\left(t_{1}\right)\right] \psi(y) d t_{1},
$$

which, after integration by parts and a change of variables, yields the D'Alembert formula

$$
u(y, t)=\frac{1}{2}[\varphi(y+t)+\varphi(y-t)]+\frac{1}{2} \int_{y-t}^{y+t} \psi(z) d z
$$

4. Let us look again at the multiple-reflection interpretation, given in Sec. 2, of the perturbation series. The reader is no doubt reminded of Bremmer's elucidation [2] of the WKB approximation for the ordinary differential equation

$$
\left(d^{2} u / d x^{2}\right)+k^{2}(x) u=0 .
$$

Bremmer's laminated medium approximation becomes very complicated when applied directly to the partial differential equation (1.1), so we indicate the connection by using the methods of this paper to derive Bremmer's results. Put

$$
\begin{aligned}
& u_{\mathrm{F}}(x)=\frac{1}{2}\left[k^{1 / 2}(x) u(x)-i k^{-1 / 2}(x) u^{\prime}(x)\right], \\
& u_{\mathrm{B}}(x)=\frac{1}{2}\left[k^{1 / 2}(x) u(x)+i k^{-1 / 2}(x) u^{\prime}(x)\right],
\end{aligned}
$$


and let $v(x)=\left(\begin{array}{l}u_{\mathrm{F}}(x) \\ u_{\mathrm{B}}(x)\end{array}\right)$. Then (4.1) becomes

$$
\frac{d v(x)}{d x}=M(x) v(x)
$$

where

$$
\begin{aligned}
M(x) & =M_{0}(x)+K(x) \\
M_{0}(x) & =\left(\begin{array}{cc}
i k(x) & 0 \\
0 & -i k(x)
\end{array}\right), \quad K(x)=\left(\begin{array}{cc}
0 & \kappa(x) \\
\kappa(x) & 0
\end{array}\right)
\end{aligned}
$$

and

$$
2 \kappa(x)=k^{\prime}(x) / k(x)
$$

Putting $V(x, s)$ for the operator which maps any solution $v$ evaluated at $s$ into its value at $x$ :

$$
V(x, s) v(s)=v(x)
$$

we see that $V(x, s)$ satisfies

$$
\frac{d}{d x} V(x, s)=M(x) V(x, s), \quad V(x, x)=1
$$

Now let

$$
\begin{gathered}
\tau(x, s)=\exp \left|i \int_{s}^{x} k(z) d z\right| \\
U(x, s)=\left(\begin{array}{cc}
\tau(x, s) & 0 \\
0 & \tau(x, s)
\end{array}\right)
\end{gathered}
$$

Then $U(x, s)$ satisfies

$$
\frac{d}{d s} U(x, s)=-U(x, s) M_{0}(x),
$$

An easy generalization of the perturbation formula (2.8) gives

$$
V(x, 0)=U(x, 0)+\int_{0}^{x} U(x, s) K(s) V(s, 0) d s .
$$

Iterating this equation and applying the resulting series to the initial datum

we get

$$
v(0)=\left(\begin{array}{l}
u_{\mathrm{F}}(0) \\
u_{\mathrm{B}}(0)
\end{array}\right)
$$

$$
\begin{aligned}
& u_{\mathrm{F}}(x)=\tau(x, 0) u_{\mathrm{F}}(0)+\int_{0}^{x} \tau(x, s) \kappa(s) \overline{\tau(s, 0)} u_{\mathrm{B}}(0) d s+\cdots \\
& u_{\mathrm{B}}(x)=\overline{\tau(x, 0)} u_{\mathrm{B}}(0)+\int_{0}^{x} \overline{\tau(x, s)} \kappa(s) \tau(s, 0) u_{\mathrm{F}}(0) d s+\cdots
\end{aligned}
$$


For $u_{\mathrm{F}}(0)=k^{1 / 2}(0) u(0), u_{\mathrm{B}}(0)=0$, we have

$$
\begin{aligned}
& u_{0}(x)=\left(\frac{k(0)}{k(x)}\right)^{1 / 2} \exp \left|\int_{0}^{x} k(z) d z\right| u(0) \\
& u_{1}(x)=\left(\frac{k(0)}{k(x)}\right)^{1 / 2} \int_{0}^{x} \exp \left|i \int_{x}^{s} k(z) d z\right| \frac{k^{\prime}(s)}{2[k(s)]^{1 / 2}} u_{0}(s) d s,
\end{aligned}
$$

and so on, giving the successive terms in Bremmer's corrections to the WKB approximation (4.16).

5. A curve $\{v(t):-\infty<t<\infty\}$ in a Hilbert space is said to be a helix if the distance $\|v(t)-v(s)\|$ between any pair $v(s), v(t)$ of points on the curve depends only on the difference $t-s$; that is, there is a function $F(t)$, called a screw-function, such that

$$
\|v(t)-v(s)\|=F(t-s)
$$

for all $s, t$. It follows from the fact that the operator $L$ in (1.11) is skew-adjoint that the phase-space trajectories corresponding to solutions of the initial-value problem for the wave-equation (1.1) are helices. In fact, we have

$$
F(t)=2^{1 / 2}[\langle v, v\rangle-\langle V(t) v, v\rangle]^{1 / 2}
$$

as the screw-function for the helix

$$
v(t)=V(t) v, \quad V(t)=e^{t L} .
$$

The possible screw-functions for helices in Hilbert space were characterized by von Neumann and Schoenberg [3]. For a phase-space of finite even dimension, every helix is a product of circles and every screw-function has a representation

$$
F^{2}(t)=\sum_{k=1}^{n} c_{k}^{2} \sin ^{2} v_{k} t
$$

the circles are the normal modes, the $v_{k}$ are the normal frequencies. In general, a helix $\{v(t):-\infty<t<\infty\}$ in a Hilbert space $\mathfrak{i}$ can be represented as

$$
v(t)=v(0)+t w+(V(t)-1) v
$$

where

$$
V(t)=e^{t L}
$$

is a one-parameter group of orthogonal transformations (so that $L$ is skew-adjoint) and the vector $w$ is invariant under $V(t)$; this was proved by von Neumann and Schoenberg [3]. In finite-dimensional phase-space, the normal modes are circles lying in the invariant subspaces of $L$. In the infinite-dimensional case, finite-dimensional invariant subspaces need not exist. For example, the operator $L_{0}$ corresponding to the wave-equation

$$
u_{t t}=u_{x x}
$$

for a homogeneous medium has precisely two irreducible invariant subspaces: the forward subspace and the backward subspace. The generalization of normal mode which is appropriate for infinite-dimensional phase-space is the irreducible helix: a helix lying entirely in an irreducible invariant subspace for the skew-adjoint operator $L$. The method 
described in this paper represents the phase-space trajectory for the heterogeneous waveequation (1.1) as a pair of coupled helices.

I wish to acknowledge my indebtedness to my colleague, Professor J. L. Synge, who discovered (by the direct calculation of successive curvatures) that the phase-space trajectory for the heterogeneous wave-equation is a helix and who pointed out the importance of the decomposition into forward and backward subspaces. The present method evolved after many discussions with him.

\section{REFERENCES}

[1] J. L. Synge, On the vibrations of a heterogeneous string, Quart. Appl. Math. (to appear)

[2] H. Bremmer, The WKB approximation as the first term of a geometric-optical series, Comm. Pure Appl. Math. 4, 105-116 (1951)

[3] J. von Neumann and I. J. Schoenberg, Fourier integrals and metric geometry, Trans. Amer. Math. Soc. 50, 226-251 (1941)

[4] T. Kato, Perturbation theory for linear operators, Springer, New York, 1961 